\title{
Efficacy of Oral Care Products Containing Copper and Zinc Salts on Oral Malodor
}

\author{
Wonkyung Choi ${ }^{1}$, Won Kyung Lee ${ }^{1}$, Won Chan Kim ${ }^{1}$, Soon Ran Song ${ }^{2}$, Ji Eun Yang ${ }^{2}$, Hyeongman Kim ${ }^{3}$, \\ Jeong Rae Lee ${ }^{4}$ \\ ${ }^{1}$ Deodorizing Tech Project, LG H\&H Research Center, Daejeon, Korea \\ ${ }^{2}$ Preventive Dentistry Research Team, LG H\&H research center, Seoul Korea \\ ${ }^{3}$ Microbiology Research Lab, LG H\&H Research Center, Daejeon, Korea \\ ${ }^{4}$ Household \& Daily Beauty Research Center, LG H\&H Research Center, Seoul, Korea
}

Objectives: The aim of this study is to investigate the efficacy of Copper and Zinc salts in suppressing and reducing oral malodor by performing in-vitro study utilizing various oral bacteria, and pilot-scale clinical study using oral care products.

Methods: Odor suppression of $\mathrm{Cu}$ and $\mathrm{Zn}$ salts in oral bacteria such as $S$. mutans, $P$. gingivalis and F. nucleatum was studied in in-vitro scale utilizing head space vials (Agilent Tech., USA) and oral chroma (Nissha FIS, Inc., Japan). Further, pilot-scale clinical investigations were conducted in double-blind, crossover-group design that was approved by LG Household and Healthcare Institutional Review Board (IRB) using toothpaste or mouthwash containing $\mathrm{Cu}$ and $\mathrm{Zn}$ salts. The subjects with morning breath were instructed to brush their teeth with toothpaste (LGHH, Korea) or use mouthwash (LGHH, Korea) containing $\mathrm{Cu}$ and $\mathrm{Zn}$ salts. The baseline and after-use oral malodor of subjects were evaluated with halimeter (Interscan, Germany), and calibrated judges using Rosenberg scale (Rosenberg, 1991).

Results: In in-vitro study, greater odor suppression was observed when treated with dual salts, compared to that treated with $\mathrm{Cu}$ or $\mathrm{Zn}$ salt alone in S. mutans, P. gingivalis and F. nucleatum. In particular, the synergistic effect of $\mathrm{Cu}$ and $\mathrm{Zn}$ in odor suppression was shown in $P$. gingivalis. In addition, in two clinical studies, both toothpaste group and mouthwash group containing $\mathrm{Cu}$ and Zn salts showed statistically significant reduction in oral malodor from the baseline of more than $40 \%$ in terms of organoleptic intensity and volatile sulfur compounds (VSCs).

Conclusions: In conclusion, the results of in-vitro study support that Copper and Zinc salts help inhibiting the growth of oral bacteria producing VSCs and eliminating VSCs fastly, and those of clinical studies exhibit that oral care products formulated with Copper and Zinc salts are effective in reducing oral malodor.

Keywords: Halitosis, Oral Malodor, Volatile Sulfur Compounds (VSC)

Copyright (C) 2021. Korean Academy of Preventive Dentistry. All rights reserved.

This is an Open Access article distributed under the terms of the Creative Commons Attribution Non-Commercial License (http://creativecommons.org/licenses/ by-nc/4.0) which permits unrestricted non-commercial use, distribution, and reproduction in any medium, provided the original work is properly cited. 